Vol. 48 (1993) [13-22]

\title{
ENLARGEABLE BANACH-LIE ALGEBRAS AND FREE TOPOLOGICAL GROUPS
}

\author{
Vladimir G. Pestov
}

We characterise in terms of free topological groups those Banach-Lie algebras with finite-dimensional centre coming from Lie groups.

\section{INTRODUCTION}

A Banach-Lie algebra $\mathfrak{g}$ is called enlargeable if it comes from a Banach-Lie group $[6,10,20-23,24,25]$. The Lie-Cartan theorem [4] says that finite-dimensional Lie algebras are enlargeable; a similar statement is no longer true for infinite-dimensional Banach-Lie algebras $[\mathbf{2 4}, \mathbf{2 0}-\mathbf{2 3}]$. There exist various criteria of enlargeability, mainly in cohomological terms $[24,23,7]$.

It seems that the question on existence of a "natural" (that is, functorial) proof of the Lie-Cartan theorem, which would be independent of both known proofs (the cohomological one by Cartan [4] and the representation-theoretic one by Ado [1]), is still open; for a discussion see [18]. In this note we reshape criteria for enlargeability of a given Banach-Lie algebra $\mathfrak{g}$ in terms of closedness of a certain subgroup of the free topological group, $F(\mathfrak{g})$, over $\mathfrak{g}$; this is done with an idea of paving a way towards a conjectural "direct" proof of the Lie-Cartan theorem.

Let $\mathfrak{g}$ be a Banach-Lie algebra. To be precise, by this term we understand a Lie algebra endowed with a complete submultiplicative norm. The Hausdorff series $H(x, y)$ converges for all $x, y$ from a sufficiently small neighbourhood of zero, $U$. The resulting binary operation $(x, y) \mapsto x . y$ makes $U$ into a local analytic Lie group, and therefore a Lie group germ, $\operatorname{loc}(\mathfrak{g})$ (in the sense of $[19,23]$ ), associated to $\mathfrak{g}$, comes into being; the functorial nature of the correspondence $\mathfrak{g} \mapsto \operatorname{loc}(\mathfrak{g})$ (which turns out to be an equivalence of categories) is well known $[3,24,20]$.

According to the Swierczkowski's Theorem on Extension of Analytic Structure [20], if a local Banach-Lie group $U$ can be embedded into a topological group $G$ as a local topological subgroup, then $G$ can be given a structure of an analytical Banach-Lie

\section{Received 16th July 1992}

This investigation was stimulated by the paper [8] by Elyahu Katz (which the author studied in 1983) and partially supported by 1992 research grant V212/451/ RGNT/594/153 from the Internal Grant Committee of the Victoria University of Wellington.

Copyright Clearance Centre, Inc. Serial-fee code: 0004-9729/93 \$A2.00+0.00. 
group extending the structure of the original local Lie group. Therefore, the problem of enlarging a Banach-Lie algebra is reduced to the problem of embedding the corresponding local Lie group into a topological group as a local topological subgroup. Ever since the paper [11] by Mal'cev it has been known that not every local topological group admits extension to a global group; the same is true relative to local Banach-Lie groups $[24,22,23]$.

It is natural to consider a universal morphism, $i_{g}$, from a Lie group germ, $g$, to a topological group, say $G_{g}$; this mapping is a morphism of group germs such that any other morphism $f: g \rightarrow G$ of this kind, where $G$ is a topological group, factors through $i_{g}$ uniquely, that is, for some unique continuous homomoprhism $\hat{f}: G_{g} \rightarrow G$ one has $i_{g} \circ \hat{f}=f$. This construction is but one example of what in category theory is referred to as universal arrows, see [13]. From Świerczkowski's theorem the following result is an easy corollary. (We shall write $i_{\mathfrak{g}}$ instead of $i_{l o c(g)}$, and $G_{\mathfrak{g}}$ instead of $G_{l o c(\mathfrak{g})}$.)

THEOREM 1. A Banach-Lie algebra $g$ is enlargeable if and only if the restriction of the universal morphism $i_{\mathfrak{g}}$ to an appropriately small local Lie group $V$ representative of the Lie group germ $l o c(g)$ is one-to-one. In this case the topological group $G_{\mathfrak{g}}$ can be given the structure of a simply connected Banach-Lie group in such a way that the restriction of the universal morphism $i_{\mathfrak{g}}$ to $V$ is a local diffeomorphism.

The universal character of the construction of $i_{g}$ makes it a very convenient but highly non-constructive device. We shall show that this construction can be performed by means of free topological groups, and it leads to a new criterion of enlargeability for Banach-Lio algebras with finite-dimensional centre in terms of the closedness of a certain subgroup of the free topological group over the underlying topological space of $\mathfrak{g}$ (Theorem 8). This criterion fails in the case where $\operatorname{dim} \mathfrak{c}(\mathfrak{g})=+\infty$ (Example). In order to give an independent proof of an auxiliary result on the structure of the topological group $G_{\mathfrak{g}}$ (Theorem 5), which can be also deduced from earlier results by Świerczkowski and van Est [21], we invoke another kind of universal arrows - the free Banach-Lie algebras [17].

We prove in passing the following curious result: a Banach-Lie algebra is enlargeable if and only if all its separable Banach-Lie subalgebras are (Theorem 7).

Since the structure of free topological groups $F\left(\mathbb{R}^{n}\right)$ is well understood $[12,9]$, then there is some hope that a "direct" proof of the Lie-Cartan theorem is within reach.

\section{MAIN CONSTRUCTION}

If $X$ is a Tychonoff topological space then by $F(X)$ we denote the free topological group over $X$ in the sense of Markov. (Our construct makes sense for free topological groups in the sense of Graev as well.) For an overview of the theory of free topological groups and a relevant bibliography, see $[2,5,9,12]$. 
Let $\mathfrak{g}$ be an arbitrary Banach-Lie algebra. Fix a neighbourhood of zero, $U$, which is "small" in the following sense:

(*) the Hausdorff series $H(x, y)$ converges for every $x, y \in U$.

(For example, $U$ may be an open or closed ball of radius less than $(1 / 3) \log (3 / 2)$ [3].) Denote by $\mathcal{N}_{\mathfrak{g}}$ a normal subgroup generated by all elements of the form $x^{-1}[x .(-y)] y, x, y \in U$. The following statement admits a straightforward verification.

ASSERTION 1. The subgroup $\mathcal{N}_{\mathrm{B}}$ does not depend on the particular choice of a neighbourhood $U$ with property (*).

Denote by $G_{\mathfrak{g}}$ the topological group quotient of $F(\mathfrak{g})$ by $\mathcal{N}_{\mathfrak{g}}$, and by $\phi_{\mathfrak{g}}: \mathfrak{g} \rightarrow G_{\mathfrak{g}}$ the restriction of the quotient homomorphism $\pi_{g}: F(\mathfrak{g}) \rightarrow G_{\mathfrak{g}}$ to $\mathfrak{g}$. Let $i_{\mathfrak{g}}$ stand for the morphism of group germs from $l o c(\mathfrak{g})$ to $G_{\mathfrak{g}}$ of which a representative is $\phi_{\mathfrak{g}}$.

THEOREM 2. The pair $\left(i_{\mathfrak{g}}, G_{\mathfrak{g}}\right)$ is a universal morphism for group germ morphisms from the Lie group germ associated to a Banach-Lie algebra $\mathfrak{g}$ to topological groups. In other words, if $G$ is a topological group (not necessarily Hausdorff) and $i: \operatorname{loc}(\mathfrak{g}) \rightarrow G$ is a morphism of group germs, then there exists a unique continuous homomorphism $\tilde{i}: G_{\mathfrak{g}} \rightarrow G$ making the diagram commutative:

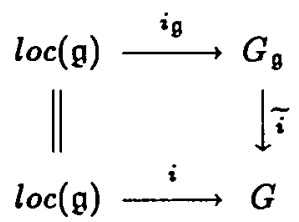

Proof: Let $U$ be an open convex neighbourhood of zero in $\mathfrak{g}$, a local Lie group representative of the Lie group germ $\operatorname{loc}(g)$. Then the morphism $i$ may be thought of as a continuous local group homomorphism from $U$ to $G$. For every $x \in \mathfrak{g}$ there is an $n \in \mathbb{N}$ such that $x / n$ is in $U$; by putting $i(x)={ }_{\text {def }}[i(x / n)]^{n}$ one obtains a mapping from the whole of $\mathfrak{g}$ to the group $G$ which we shall still denote by $i$. It does not depend on the choice of $n$ and is continuous at every point $x \in \mathfrak{g}$ as a composition of three continuous mappings: $x \mapsto x / n \mapsto i(x / n) \mapsto[i(x / n)]^{n}$, if the number $n$ has been chosen uniformly in a neighbourhood of $x$. It extends to a continuous homomorphism of topological groups $\widehat{i}: F(g) \rightarrow G$. Obvisouly, the kernel of $\widehat{i}$ contains the subgroup $\mathcal{N}_{\mathfrak{g}}$ and therefore $\hat{i}$ factors through $\pi_{\mathfrak{g}}$ giving rise to a continuous homomorphism $\widetilde{i}: G_{\mathfrak{g}} \rightarrow G$. Since clearly $\widehat{i} \mid U=i$ then the above diagram commutes.

ThEOREM 3. The following conditions are equivalent for a Banach-Lie aglebra g.

(i) $\mathrm{g}$ is enlargeable; 
(ii) the intersection $\mathcal{N}_{\mathfrak{g}} \cap \mathfrak{g}$ is discrete in $\mathfrak{g}$;

(iii) the restriction of $\phi_{\mathfrak{B}}$ to a neighbourhood of zero in $\mathfrak{g}$ is one-to-one;

(iv) the topological group $G_{\mathrm{g}}$ can be given a structure of an analytical BanachLie group in such a way that $\phi_{\mathfrak{g}}$ is a local analytical diffeomorphism. In this case Lie $\left(G_{\mathfrak{g}}\right) \cong \mathfrak{g}, \phi_{\mathfrak{g}}=\exp _{G_{\mathfrak{g}}}$, and $G_{\mathfrak{g}}$ is simply connected.

Proof: $(i) \Rightarrow($ iii $)$ : Let $G$ be a simply connected Banach-Lie group associated to $\mathfrak{g}$, and let $\exp _{G}$ be the corresponding exponential mapping. Since obviously $\mathcal{N}_{\mathfrak{g}} \subset$ $\left\{x \in \mathfrak{g}: \exp _{G}(x)=e_{G}\right\}$, then for all $x, y$ in a small enough neighbourhood of zero in $\mathfrak{g}$ one has: if $\phi_{\mathfrak{g}}(x)=\phi_{\mathfrak{g}}(y)$ then $\exp _{G}(x)=\exp _{G}(y)$. Since $\exp _{G}$ is locally one-to-one, then so is $\phi_{g}$.

(ii) $\Leftrightarrow($ iii): an immedeate check.

$($ iii $) \Rightarrow($ iv $)$ : see Theorem 1 .

(iv) $\Rightarrow(i)$ : obvious.

As a matter of fact, all these characterisations were discovered decades ago, perhaps in a different form $[4,24,20-23,25,6]$. For example, the set $\mathcal{N}_{\mathfrak{g}} \cap \mathfrak{g}$ forms an additive subgroup of $\mathfrak{g}$ isomorphic to the period group $\operatorname{Per}(\mathfrak{g})$ of the Lie algebra $\mathfrak{g}[\mathbf{2 4}]$. What is new, is our suggestion to consider topology on the group $F(\mathfrak{g})$. Traditionally, the abstract free group over an arbitrarily small neighbourhood of zero in $\mathfrak{g}$ was given full attention (see $[\mathbf{2 2}, \mathbf{2 3}])$ rather than the free topological group over the Lie algebra $\mathfrak{g}$. Free topological groups over neighbourhoods of the identity in finite-dimensional Lie groups were first considered in [8].

If a Banach-Lie algebra $\mathfrak{g}$ is enlargeable, then the subgroup $\mathcal{N}_{\mathfrak{g}}$ of the free topological group $F(\mathfrak{g})$ is easily checked to be closed; indeed, it is the kernel of the homomoprhism $\pi_{g}: F(g) \rightarrow G_{g}$, and according to Theorem 2 and item (iv) of Theorem 3, $\pi_{\mathfrak{g}}$ is continuous while $G_{\mathfrak{g}}$ is endowed with a Hausdorff topology.

It turns out that the closedness of $\mathcal{N}_{\mathfrak{g}}$ is not sufficent for enlargeability of $\mathfrak{g}$, as the following example shows.

EXAMPLE. Let $g$ be a Banach-Lie algebra with the following properties.

(a) the extension

$$
0 \rightarrow \mathfrak{c}(\mathfrak{g}) \rightarrow \mathfrak{g} \rightarrow \mathfrak{g} / \mathfrak{c}(\mathfrak{g}) \rightarrow 0
$$

is topologically split $(\mathfrak{c}(\mathfrak{g})$ is the centre of $\mathfrak{g})$;

(b) $\mathfrak{g}$ is enlargeable, and the centre of the corresponding connected simply connected Banach-Lie group $G$ contains a circle, $T$.

For examples of such Lie algebras, see $[24,25,3,6]$. Fix a submultiplicative norm on $\mathfrak{g}$ and define for every $n=1,2, \ldots$ a submultiplicative norm $\|\cdot\|_{n}$ on $\mathfrak{g}$ by

$$
\|x+y\|_{n}=d_{\text {def }}\|x\|_{\mathfrak{g} / \mathfrak{c}(\mathfrak{g})}+\|y\|_{\mathfrak{c}(\mathfrak{g})}(x \in \mathfrak{g} / \mathfrak{c}(\mathfrak{g}), y \in \mathfrak{c}(\mathfrak{g}))
$$


Denote by $g_{n}$ the Lie algebra $g$ with the norm $\|\cdot\|_{n}$, and by $g_{\infty}$ the $l_{1}$-type sum of the Lie algebras $\mathfrak{g}_{n}$, that is, the completion of the direct sum $\oplus_{n=1}^{\infty} \mathfrak{g}_{n}$ with respect to the submultiplicative norm

$$
\left\|\left(x_{n}\right)_{n \in \mathbf{N}}\right\|=_{\text {def }} \sum_{n \in \mathbf{N}}\left\|x_{n}\right\|_{n}
$$

Denote for every $n=1,2, \ldots$ by $p_{n}$ the canonical projection $\mathfrak{g}_{\infty} \rightarrow \mathfrak{g}_{n}$, and by $\hat{p}_{n}$ its continuous homomorphic extension $F\left(\mathfrak{g}_{\infty}\right) \rightarrow F\left(\mathfrak{g}_{n}\right)$. We shall show now that

$$
\mathcal{N}_{\mathfrak{g}_{\infty}}=\bigcap_{n=1}^{\infty}{\widetilde{p_{n}}}^{1}\left(\mathcal{N}_{\mathfrak{g}_{n}}\right)
$$

Since all the Lie subalgebras $\mathfrak{g}_{n}$ of $\mathfrak{h}$ commute with each other, then (1) is derived from the following simple observation: the subgroup $\mathcal{N}_{\mathbf{g}_{\infty}}$ is generated by a subset with the property (*) of the form $\bigcap_{n=1}^{\infty}{\widetilde{p_{n}}}^{-1}\left(U_{n}\right)$; just pick for every $n$ an $U_{n} \subset \mathfrak{g}_{n}$ satisfying (*).

The property (1) implies that the subgroup $\mathcal{N}_{\mathrm{B}_{\infty}}$ is closed. Another corollary of (1) is the fact that the period group $\operatorname{Per}\left(g_{\infty}\right) \cong F\left(g_{\infty}\right) / \mathcal{N}_{g_{\infty}}$ of the Lie algebra $g_{\infty}$ is the (completed) infinite direct sum of circles $T_{n}$ sitting in the centres of the Lie algebras $\mathfrak{g}_{n}$. Since the radii of those circles approach zero as $n \rightarrow \infty$ then the group $P e r\left(\mathfrak{g}_{\infty}\right)$ is non-discrete and therefore $G_{g_{\infty}}$ is non-enlargeable.

However, things are different for Banach-Lie algebras $\mathfrak{g}$ with finite-dimensional centre, $\mathfrak{c}(\mathfrak{g})$, in which case the discreteness of the interesection $\mathcal{N}_{\mathfrak{g}} \cap \mathfrak{g}$ can be deduced from closedness of $\mathcal{N}_{\mathfrak{g}}$ in $F(\mathfrak{g})$.

Lemma 1. Let $V$ be a closed local subgroup of a finite-dimensional local Lie group $U$ such that the intersections of $V$ with all one-parameter Lie subgroups are discrete. Then $V$ is discrete.

ProOF: Obviously follows from compactness of a unit sphere in a finite-dimensional Euclidean space.

Theorem 4. Let $\mathfrak{g}$ be a Banach-Lie algebra. Then $\mathcal{N}_{\mathfrak{g}} \cap \mathfrak{g} \subset \mathfrak{c}(\mathfrak{g})$.

Proof: Actually, this is a classical observation, and the proof of it is "functorial" (see [24]). It stems from the fact that the group $\mathcal{N}_{\mathrm{g}}$ is contained in the (closed!) kernel of the continuous homomorphism $a d_{\mathfrak{g}} \widehat{\exp _{A u t} \mathfrak{g}}: G_{\mathfrak{g}} \rightarrow$ Aut $\mathfrak{g}$ where $a d_{\mathfrak{g}}$ is the adjoint representation $\mathfrak{g} \rightarrow \operatorname{Der} \mathfrak{g}$. Therefore, $\mathcal{N}_{\mathfrak{g}} \cap \mathfrak{g} \subset$ ker $\operatorname{ad}_{\mathfrak{g}}=\mathfrak{c}(\mathfrak{g})$.

THEOREM 5. Let $\mathfrak{g}$ be a separable Banach-Lie algebra. Then $\mathcal{N}_{\mathfrak{g}} \cap \mathfrak{g}$ contains no one-parameter local subgroups of $\operatorname{loc}(\mathfrak{g})$.

Proof: First of all, we shall recall some of our earlier results [17]. 
Theonem 6. Let $E$ be a normed space. There exist a complete normed Lie algebra $\mathcal{F} \mathcal{L}(E)$ and a linear isometrical embedding $i_{E}: E \hookrightarrow \mathcal{F} \mathcal{L}(E)$ with the following properties.

(1) $i_{E}(E)$ topologically generates $\mathcal{F} \mathcal{L}(E)$.

(2) For an arbitrary complete normed Lie algebra $\mathcal{L}$ and any linear operator $f: E \rightarrow \mathcal{L}$ of norm $\leqslant 1$, there exists a Lie algebra homomorphism $\widehat{f}: \mathcal{F} \mathcal{L}(E) \rightarrow \mathcal{L}$ of norm $\leqslant 1$ such that $\hat{f} \circ i_{E}=f$.

The pair $\left(\mathcal{F} \mathcal{L}(E), i_{E}\right)$ with the properties (1) and (2) is essentially unique.

The Banach-Lie algebra $\mathcal{F} \mathcal{L}(E)$ is termed the free Banach-Lie algebra over a normed space $E$. It turns out that if $\operatorname{dim} E \geqslant 2$ then $\mathcal{F} \mathcal{L}(E)$ is centreless and therefore, for any normed space $E$, the free Banach-Lie algebra $\mathcal{F L}(E)$ is enlargeable, the corresponding Banach-Lie group in the case $\operatorname{dim} E \geqslant 2$ being a Banach-Lie subgroup of the automorphism group of $\mathcal{F C}(E)$ generated by the image of the latter Lie algebra under the exponential mapping. As a result, every Banach-Lie algebra, $\mathfrak{g}$, is a quotient algebra of an enlargeable Banach-Lie algebra, and in a "functorial way" indeed: the identity mapping $i d_{\mathfrak{g}}$ from $\mathfrak{g}$ to its underlying Banach (= complete normed) space extends to a quotient Banach-Lie algebra homomorphism $\widehat{i d_{\mathfrak{g}}}: \mathcal{F} \mathcal{L}(\mathfrak{g}) \rightarrow \mathfrak{g}$, which is easily verified to be an open Lie algebra morphism onto. The Banach-Lie algebra $\mathcal{F} \mathcal{L}(E)$ is separable if and only if $E$ so is.

Now suppose $\mathfrak{g}$ is a separable Banach-Lie algebra. Denote the kernel of $\widehat{i d_{\mathfrak{g}}}$ by $J_{\mathfrak{g}}$; it is a closed Lie ideal. Now let $\mathcal{G}_{\mathfrak{g}}$ be a connected simply connected Banach-Lie group corresponding to the enlargeable Lie algebra $\mathcal{F} \mathcal{L}(g)$ and let $\exp _{(\mathrm{g})}$ be the exponential mapping $\mathcal{F} \mathcal{L}(\mathfrak{g}) \rightarrow \mathcal{G}_{\mathfrak{g}}$. Let $J_{\mathfrak{g}}^{\times}$stand for a subgroup in $\mathcal{G}_{\mathfrak{g}}$ algebraically generated by the image of $J_{\mathfrak{g}}$ under $\exp _{(\mathfrak{g})}$. This group is normal but not necessarily closed. Denote by $\exp g$ the quotient topological group $\mathcal{G}_{\mathfrak{g}} / J_{\mathfrak{g}}^{\times}$.

A natural continuous mapping $\exp _{\mathfrak{g}}: \mathfrak{g} \rightarrow \exp \mathfrak{g}$ is obtained by factoring the exponential map $\exp _{(\mathfrak{g})}: \mathcal{F} \mathcal{L}(\mathfrak{g}) \rightarrow \mathcal{G}_{\mathfrak{g}}$ through $\widehat{i d}_{\mathfrak{g}}$. In view of the universality of the mapping $i_{g}$ (Theorem 2), it suffices to show that for an arbitrary $x \in g \backslash\{0\}$, the image under the mapping $\exp _{\mathfrak{g}}$ of the one-dimensional linear space spanned by $x$ is non-degenerate in $\exp \mathfrak{g}$, that is, for an arbitrary $y \in \mathcal{F} \mathcal{L}(\mathfrak{g}) \backslash J_{\mathfrak{g}}$, the one-parameter subgroup of $\mathcal{G}_{g}$ tangent to $y$ is not entirely contained in the subgroup $J_{\mathfrak{g}}^{\times}$. But this follows from [3, Chapter III, Section 6.2 Corollary 2].

COROLlary 1. Let $\mathfrak{g}$ be a separable Banach-Lie algebra such that the subgroup $\mathcal{N}_{\mathrm{g}}$ is closed in $F(\mathrm{~g})$. Then the intersection of $\mathcal{N}_{\mathfrak{g}}$ with every one-dimensional local subgroup (one-dimensional linear subspace) of $\mathrm{g}$ is discrete.

Proof: Every proper closed subgroup of $\mathbb{R}$ is discrete; now apply Theorem 5 .

Corollary 2. Let $\mathfrak{g}$ be a separable Banach-Lie algebra with finite-dimensional 
centre. Then $\mathfrak{g}$ is enlargeable if and only if the subgroup $\mathcal{N}_{\mathfrak{g}}$ is closed in $F(\mathfrak{g})$. In this case the quotient topological group $G_{g}$ carries a natural structure of a Banach-Lie group associated to $\mathrm{g}$.

The separability restriction is removed with the help of the following curious result, which is also new and of interest by itself.

THEOREM 7. A Banach-Lie algebra $\mathfrak{g}$ is enlargeable if and only if every separable Banach-Lie subalgebra of $\mathfrak{g}$ is enlargeable.

Proof: Denote by $\mathfrak{H}$ the family of all separable Banach-Lie subalgebras of $\mathfrak{g}$ partially ordered by inclusion. Obviously, $\cup \mathfrak{H}=\mathfrak{g}$. One can assume that $\mathfrak{g}$ is inseparable and therefore $\mathfrak{H}$ has no upper bound. For every $\mathfrak{h} \in \mathfrak{H}$ denote by $G_{\mathfrak{h}}$ a simply connected Lie group associated to $\mathfrak{h}$ and by $\exp _{\mathfrak{h}}: \mathfrak{h} \rightarrow G_{\mathfrak{h}}$ the corresponding exponential mapping. Put for each $\mathfrak{h} \in \mathfrak{H}$

$$
\varepsilon(\mathfrak{h})={ }_{\text {def }} \min \left\{1, \max \left\{r \in \mathbb{R}_{+}: \exp _{\mathfrak{h}} \mid B_{r}(0) \text { is one-to-one }\right\}\right\}
$$

where $B_{r}(0)$ is the open ball of radius $r$ centred at zero. If $\mathfrak{h}_{1} \subset \mathfrak{h}_{2}$ then the inclusion mapping $i_{\mathfrak{h}_{1}}^{\mathfrak{h}_{2}}: \mathfrak{h}_{1} \rightarrow \mathfrak{h}_{2}$ gives rise to a Lie group morphism $\hat{i}_{\mathfrak{h}_{1}}^{h_{2}}: G_{\mathfrak{h}_{1}} \rightarrow G_{\mathfrak{h}_{2}}$ which commutes with the exponential mappings. One concludes that if $\mathfrak{h}_{1} \subset \mathfrak{h}_{2}$ then $\varepsilon\left(\mathfrak{h}_{1}\right) \geqslant$ $\varepsilon\left(\mathfrak{h}_{2}\right)$, and therefore $\varepsilon$ is a non-increasing mapping from the directed partially ordered set $\mathfrak{H}$ to $\mathbb{R}$. Put $\varepsilon_{0}=\inf \{\varepsilon(\mathfrak{h}): \mathfrak{h} \in \mathfrak{H}\}$. Clearly, $\varepsilon_{0} \geqslant 0$, and the case $\varepsilon_{0}=0$ is impossible because $\mathfrak{H}$ contains unions of countable subfamilies. Therefore, for every $\mathfrak{h} \in \mathfrak{H}$, the restriction of the exponential mapping $e x p_{\mathfrak{h}}$ to the open ball of radius $\varepsilon_{0}>0$ about zero is one-to-one. Now we apply the Local Theorem on Enlargeability of Banach-Lie Algebras, $[15,16]$.

Finally we come to the central

THEOREM 8. A Banach-Lie algebra $\mathfrak{g}$ with finite-dimensional center is enlargeable if and only if the subgroup $\mathcal{N}_{\mathfrak{g}}$ is closed in $F(\mathfrak{g})$. In this case the quotient topological group $G_{\mathfrak{g}}$ carries a natural structure of a Banach-Lie group associated to $\mathfrak{g}$.

Proof: Necessity was pointed out at just before our Example, and sufficiency follows from Corollary 2 and Theorem 7 .

\section{Final Discussion}

1. All the structures participating in our construct come into being in a functorial 
way as the objects of the following commutative diagram

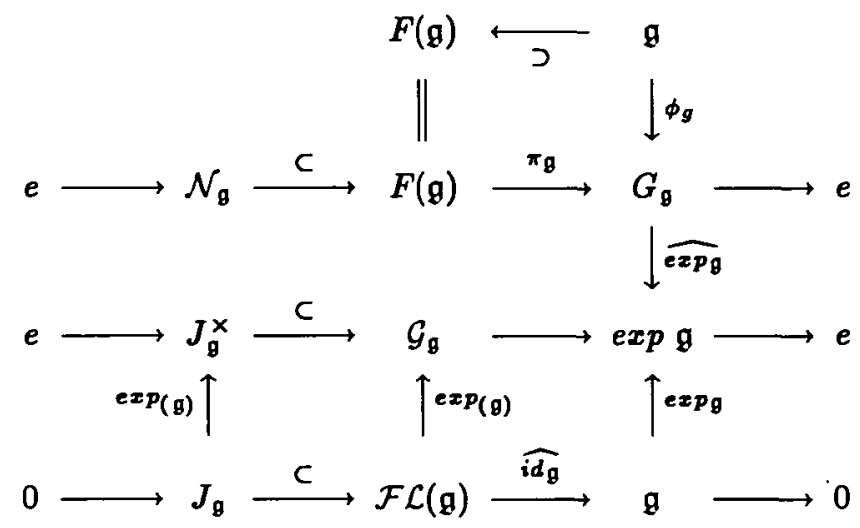

(The arrow $\mathfrak{g} \stackrel{=}{\longrightarrow} \mathfrak{g}$ is understood.)

A direct proof of the Lie-Cartan theorem would result from applying corollary 2 to a finite-dimensional Banach-Lie algebra, $\mathfrak{g}$. The only missing link is the verification of closedness of $\mathcal{N}_{\mathfrak{g}}$ in $F(\mathfrak{g})$ which should rely solely on topological structure of the free topological group $F(g)$. Since the structure of free topological groups over $\boldsymbol{k}_{\boldsymbol{\omega}}$-spaces, and in particular over $\mathbb{R}^{n}$, is deeply understood now ([12]; see also [9] and references therein), then the problem of recovering this link does not seem entirely hopeless. We conjecture that $\mathcal{N}_{\mathfrak{B}}$ is the free topological group over a $k_{\omega}$ space, and thence it is complete and closed.

2. The arguments of the kind discussed in this note are no longer solid beyond the setting of Banach-Lie algebras and groups. For example, one cannot in general expect the Hausdorff series to converge in a neighbourhood of zero of a Fréchet-Lie algebra; the exponential mapping may not be even $C^{\infty}$; moreover, it is not known yet whether every smooth Fréchet-Lie group possesses an exponential mapping, [10, 14]. In general, we anticipate the techniques of universal arrows to forgetful functors to lead to "pathological" examples of Fréchet-Lie groups rather than some positive results. Nevertheless, our construct makes perfect sense for the so-called Baker-Campbell-Hausdorff Lie groups modeled over locally convex spaces [14].

3. In connection with a result [26] that the free topological group $F\left(\mathbb{R}^{n}\right)$ is topologically a manifold modeled over the locally convex space $\mathbb{R}^{\boldsymbol{\omega}} \cong \underset{\lim }{\longrightarrow}\left\{\mathbb{R}^{k}: k \in \mathbb{N}\right\}$, one wonders whether $F\left(\mathbb{R}^{n}\right)$ can be given a structure of an (at least, $C^{1}$ ) Lie group modeled over the same $(L B)$-space $\mathbb{R}^{\omega}$. What may its Lie algebra look like?

\section{References}

[1] I.D. Ado, 'The representation of Lie algebras by matrices', Amer. Math. Soc. Transl. Ser. 19 (1962), 308-327. 
[2] A.V. Arhangel'skiř, 'Classes of topological groups', Russian Math. Surveys 36 (1981), 151-174.

[3] N. Bourbaki, Lie groups and Lie algebras (Springer-Verlag, Berlin, Heidelberg, New York, 1989).

[4] E. Cartan, La topologie des groupes de Lie (Hermann, Paris, 1936).

[5] W.W. Comfort, K.H. Hofmann and D. Remus, 'A survey on topological groups and semigroups', in General Topology - Recent Developments (North-Holland, Amsterdam, 1992) (to appear).

[6] J. Eells, jr., 'A setting for global analysis', Bull. Amer. Math. Soc. 72 (1966), 751-807.

[7] D.B. Fuks, Cohomology of infinite-dimensional Lie algebras, Translated from Russian by A.B. Sosinski (Consultants Bureau, New York, London, 1986).

[8] E. Katz, 'Free topological groups and principal fiber bundles', Duke Math. J. 42 (1975), 83-90.

[9] E. Katz and S.A. Morris, 'Free Abelian topological groups on countable CW-complexes', Bull. Austral. Math. Soc. 41 (1990), 451-456.

[10] O. Kobayashi, A. Yoshioka, Y. Maeda and H. Omori, 'The theory of infinite-dimensional Lie groups and its applications', Acta Appl. Math. 3 (1985), 71-106.

[11] A.I. Mal'cev, 'Sur les groupes topologiques locaux et complets', C.R. (Doklady) Acad. Sci. URSS (N.S.) 32 (1941), 606-608.

[12] J. Mack, S.A. Morris and E.T. Ordman, 'Free topological groups and the projective dimension of a locally compact abelian group', Proc. Amer. Math. Soc. 40 (1973), 399-402.

[13] S. Mac Lane, Categories for the working mathematician, Graduate Texts in Mathematics 5 (Springer-Verlag, Berlin, Heidelberg, New York, 1971).

[14] J. Milnor, 'Remarks on infinite-dimensional Lie groups', in Relativité, groupes et topologie II. Les Houches, Session XL (Elsevier Sci. Publ., Amsterdam, 1984), pp. 1007-1058.

[15] V.G. Pestov, 'Fermeture nonstandard des algèbres et groupes de Lie banachiques', C.R. Acad. Sci. Paris Ser. 1 Math. 306 (1988), 643-645.

[16] V.G. Pestov, 'Nonstandard hulls of Banach-Lie groups and algebras', Nova Journal of Algebras and Geometries (to appear).

[17] V.G. Pestov, 'Free Banach-Lie algebras, couniversal Banach-Lie groups, and more', Pacific J. Math. 157 (1993), 137-144.

[18] M.M. Postnikov, Lie groups and Lie algebras, Lectures in geometry. Semester V, Translated from Russian by V. Shokurov (Mir, Moscow, 1986).

[19] A. Robinson, 'Germs', in Applications of model theory to algebra, analysis and probability (Holt, Rinehart and Winston, New York, 1969).

[20] S. Swierczkowski, 'Embedding theorems for local analytic groups', Acta Math. 114 (1965), 207-235.

[21] S. Swierczkowski, 'The path-functor on Banach-Lie algebras', Indag. Math. 33 (1971), 235-239.

[22] S. Swierczkowski, 'On the cohomology of local groups', J. Austral. Math. Soc. 12 (1971), 249-255. 
[23] S. Świerczkowski, 'Cohomology of group germs and Lie algebras', Pacific J. Math. 39 (1971), 471-482.

[24] W.T. van Est and T.J. Korthagen, 'Non-enlargible Lie algebras', Nederl. Akad. Wetensch. Proc. A26 (1964), 15-31.

[25] W.T. van Est and S. Swierczkowski, 'The path functor and faithful representability of Banach Lie algebras', J. Austral. Math. Soc. 16 (1973), 471-482.

[26] M.M. Zarichnyı̌, 'Free topological groups of absolute neighbourhood retracts and infinite-dimensional manifolds', Soviet Math. Dokl. 26 (1982), 367-371.

Department of Mathematics

Victoria University of Wellington

Wellington

New Zealand

E-mail: vova@kauri.vuw.ac.nz 